\title{
A comparison of Bayesian inference and gradient-based approaches for friction parameter estimation
}

\author{
Simon C. Warder ${ }^{\mathrm{a}, *}$, Athanasios Angeloudis ${ }^{\mathrm{c}}$, Stephan C. Kramer ${ }^{\mathrm{a}}$, Colin J. Cotter ${ }^{\mathrm{b}}$, Matthew D. Piggott ${ }^{\mathrm{a}}$ \\ ${ }^{a}$ Department of Earth Science and Engineering, Imperial College London, UK \\ ${ }^{b}$ Department of Mathematics, Imperial College London, UK \\ ${ }^{c}$ School of Engineering, Institute for Infrastructure 8 Environment, University of Edinburgh, UK
}

\begin{abstract}
Numerical tidal models are essential to the study of a variety of coastal ocean processes, but typically rely on uncertain inputs, including a bottom friction parameter which can in principle be spatially varying. Here we employ an adjoint-capable numerical ocean model, Thetis, and apply it to the Bristol Channel and Severn Estuary, using a spatially varying Manning coefficient within the bottom friction parameterisation. The spatial variation in the coefficient is a priori constrained by a categorisation of the sediment type found on the sea bed into three groups: rock, sediment containing gravel, and sediment containing only sand. We compare two calibration methods to estimate the three corresponding Manning coefficients using tide gauge observation data. The first method consists of Bayesian inversion via a Markov Chain Monte Carlo algorithm, using a Gaussian process emulator as a surrogate for the full numerical model, while the second uses a gradient-based approach via the adjoint mode of the numerical model. We first apply these methods to a 'synthetic' experiment, then to the assimilation of real data; in each experiment we compare the results from each method and their respective computational cost. We further find that the use of the estimated Manning coefficients also reduces the model-observation misfit when tested within an independent numerical model, TELEMAC-2D, indicating that the calibration procedure has identified non model-specific and physically meaningful parameters.
\end{abstract}

Keywords: Parameter estimation, Bayesian inversion, MCMC, Adjoint, Gradient-based optimisation

\section{Introduction}

Numerical coastal ocean, and specifically tidal, models have a wide range of applications; studies of tidal energy systems (Neill et al., 2018) or coastal sediment transport (Xie et al., 2009) often rely directly on tidal modelling of varying complexity, while tides are also a crucial component of storm surge (Horsburgh and Wilson, 2007) and other coastal hazards. While the prediction of tides on a single location basis via tidal harmonic analysis long pre-dates numerical methods (Schureman, 1941), tidal modelling is nevertheless a

\footnotetext{
${ }^{*}$ Corresponding author

Email addresses: s.warder15@imperial.ac.uk (Simon C. Warder), a.angeloudis@ed.ac.uk (Athanasios Angeloudis), s.kramer@imperial.ac.uk (Stephan C. Kramer), colin.cotter@imperial.ac.uk (Colin J. Cotter), m.d.piggott@imperial.ac.uk (Matthew D. Piggott)
} 
central component of coastal ocean models, and much effort goes into the development and calibration of tidal models.

Tidal models can in principle rely on a number of uncertain parameters, which the user may tune in order to obtain good agreement between model outputs and observations. As is common for tidal model calibration, here our focus is on calibration with respect to a bottom friction parameter, which captures the loss of energy due to frictional effects at the sea bed and has significant influence on tidal and storm surge models (Warder et al., Submitted 2020). Since this represents a real physical process, an ideal model calibration should produce physically meaningful values for the coefficient. In reality, however, the calibration process corrects for multiple sources of error, arising from assumptions made in the underlying model equations, the discretisation and numerical solution of the equations, as well as other uncertain model inputs such as bathymetry and tidal boundary conditions. The calibration process is also influenced by potential errors in the observations.

A user seeking to calibrate a numerical tidal model must first select the number of degrees of freedom in the friction coefficient parameter space. In the simplest case, a single (spatially uniform) friction coefficient can be applied; this is the commonly taken approach for many applications, both idealised as well as realistic. The most complex possible choice is to allow the bottom friction coefficient to vary freely over the whole domain, and in this case it is common to supplement the observation data with a form of regularisation, to avoid the problem of over-fitting (Maßmann, 2010a). Intermediate complexity in the friction coefficient can be achieved via several approaches. One common approach is the so-called independent points scheme, where the friction coefficient field is determined by interpolation from a selected number of 'independent points' (Zhang et al., 2011, Chen et al., 2014), distributed uniformly or according to physical features such as the bathymetry gradient (Lu and Zhang, 2006). Another approach, used by Heemink et al. (2002), divides the domain into regions of similar influence on the model-observation misfit using an adjoint gradient-based method, also taking into account the physical properties of the model. The method we present takes a physics-based approach to simplify the friction coefficient parameter space, by dividing the model domain according to the type of sediment found on the sea bed; this is similar to the approaches of Guillou and Thiébot (2016) and Sraj et al. (2014b), and incorporates prior knowledge about the bottom friction process via grain size (and hence roughness length) data.

Once a suitable input parameter space has been selected, a suitable calibration method must be chosen to constrain the parameters based on observation data. This choice is typically related to the selected number of degrees of freedom. A standard strategy involves minimising some measure of the model-observation misfit, such that the calibration problem can be formulated as an optimisation problem. In the case of a uniform friction coefficient, a very simple (brute force) approach is possible, where the numerical model is run with a variety of coefficient values, with the optimal choice being the value which minimises the misfit (Guillou and Thiébot, 2016). This approach rapidly becomes unfeasible as the number of degrees of freedom increases, due to the computational cost of running the model with many combinations of friction parameters. For higher 
degrees of freedom, the use of adjoint-capable models (Chen et al., 2014, Lu and Zhang, 2006, Maßmann, 2010b,a, Zhang et al., 2011, Heemink et al., 2002) (or other gradient-calculation methods (Sraj et al., 2014a)) is appropriate, as they allow the gradient of the model-observation misfit to be computed efficiently with respect to an arbitrarily large set of input parameters. This gradient information permits the use of efficient gradient-based optimisation algorithms in minimising model-observation misfit. Hence this establishes the optimal choice of friction parameters, for any of the choices of friction parameter space outlined above, although issues of ill-posedness and local minima may need to be addressed. However, unless the Hessian is also computed alongside the gradient (Sraj et al., 2014a), such gradient-based methods do not provide a direct estimate of parameter uncertainty. Another frequently used approach for parameter estimation is the use of Bayesian inversion, typically via Markov Chain Monte Carlo (MCMC) methods (Hall et al., 2011, Sraj et al., 2013, 2014b). Such methods have the advantage over adjoint-based methods that they do not require the computation of the derivative of the numerical model (which can be hard to implement for complex codes (Mitusch et al., 2019)), and that they yield estimates of the uncertainty in the inferred parameters. However, they rely on large numbers of forward model runs $\left(\mathcal{O}\left(10^{4}\right)\right.$ or more e.g. Tagade et al. (2013), Pall et al. (2018), Haario et al. (2001), Sraj et al. (2014b)), and the numerical model is therefore usually substituted with a fast surrogate model such as a Gaussian process emulator (Hall et al., 2011, Tagade et al., 2013) or polynomial chaos expansion (Sraj et al., 2014b). Such surrogate models are trained using a selected number of full model runs spanning the input parameter space (or prior distribution). Statistical data assimilation using Kalman filters has also been used for friction parameter estimation (Mayo et al., 2014), but is more commonly used in the context of state estimation, or joint state and parameter estimation (Evensen, 2009), and is not considered here.

In this work, we implement and compare two methods for the calibration of bottom friction parameters via the assimilation of observation data, using the Thetis numerical coastal ocean model. The first uses a Markov Chain Monte Carlo method, via the use of a Gaussian process emulator as a surrogate for the full numerical model, and the second uses a gradient-based approach using the adjoint mode available within Thetis. The use of these two contrasting methods allows a direct comparison, both in terms of the resulting parameter estimates and the computational cost, of each method. Since calibration methods do not correct only for errors due to the friction parameterisation but also for various other modelling and discretisation errors, we also attempt to quantify the 'success' of each method in determining non model-specific friction parameters, by comparing the model-observation misfit of a second model, TELEMAC-2D, using the sets of friction parameters selected by each calibration method. To the authors' knowledge, this is the first direct comparison of adjoint gradient-based optimisation and Bayesian inversion via MCMC for parameter estimation within a coastal ocean model. The closest related study to date is by Sraj et al. (2013, 2014a), where the use of a polynomial chaos expansion for both gradient-based optimisation and Bayesian inversion via MCMC has been compared for the purposes of wind drag parameter estimation.

In Section 2 we describe the data and models used within this work, and the calibration techniques are 
described in Section 3. Results from a synthetic experiment and the assimilation of real data are described in Section 4, these results are discussed in Section 5, and we draw conclusions in Section 6.

\section{Description of data and models}

\subsection{Data}

In this work we consider the Bristol Channel and Severn Estuary region on the UK west coast as a real world case study location and use data from two sources for the purposes of model calibration and validation:

(i) 11 locations at which tidal harmonic data is available (National Oceanography Centre, personal communication 2018), which are shown as green squares in Fig. 1. To compare modelled results with these data, the model must be run for a suitably long time (ideally a month or more), and a harmonic analysis performed at these locations.

(ii) Five tide gauges where quality controlled timeseries surface elevation data is available from the British Oceanographic Data Centre (BODC). These locations are shown in Fig. 1 by red circles. At these locations, modelled surface elevations can be compared directly with observations without the need for harmonic analysis, provided that the model adequately captures any meteorological effects influencing the tide gauge signal during the comparison window, or that the meteorological conditions are sufficiently calm that their effect can be neglected.

\subsection{Thetis}

The primary model applied here is Thetis, an unstructured-mesh finite element coastal ocean model (Kärnä et al., 2018) which utilises the Firedrake finite element code generation framework (Rathgeber et al., 2016). We employ Thetis in its two-dimensional configuration (as in Vouriot et al. (2019)), which solves the nonlinear shallow water equations given by

$$
\begin{aligned}
\frac{\partial \eta}{\partial t}+\nabla \cdot(H \mathbf{u}) & =0 \\
\frac{\partial \mathbf{u}}{\partial t}+\mathbf{u} \cdot \nabla \mathbf{u}+\mathbf{F}_{\mathbf{C}}+g \nabla \eta & =-\frac{\boldsymbol{\tau}_{b}}{\rho H}+\nabla \cdot\left(\nu_{h}\left(\nabla \mathbf{u}+\nabla \mathbf{u}^{T}\right)\right),
\end{aligned}
$$

where $\eta$ is the free surface elevation, $H=\eta+h$ is the total water depth, $h$ is the bathymetry (measured positive downwards), $\mathbf{u}$ is the two-dimensional depth-averaged velocity, $\mathbf{F}_{\mathbf{C}}$ is the Coriolis force, $g$ is the acceleration due to gravity, $\rho$ is the water density, $\boldsymbol{\tau}_{b}$ is the bottom stress due to friction between the ocean and sea bed, and $\nu_{h}$ is the kinematic viscosity. Here, we parameterise the bottom friction $\boldsymbol{\tau}_{\boldsymbol{b}}$ via a Manning's $n$ formulation

$$
\frac{\boldsymbol{\tau}_{b}}{\rho}=\frac{g n^{2}}{H^{\frac{1}{3}}}|\mathbf{u}| \mathbf{u}
$$

where $n$ is the Manning coefficient (units $\mathrm{s} \mathrm{m}^{-1 / 3}$ ). In this work, and for the purposes of model calibration, the model domain is divided according to the type of sediment on the ocean bed, based on data from SHOM (Service Hydrographique et Océanographique de la Marine) (SHOM, 2019). We divide sediment 
types into three groups: (1) rock (NFRoche in the SHOM dataset), (2) sediment containing gravel (NFG, NFSG, NFSGV), and (3) sediment containing only sand (NFS, NFSV, NFV, NFVS), and assign a different Manning coefficient $\left(n_{1}, n_{2}, n_{3}\right)$ to each sediment group respectively. We choose to use three sediment groups as a cautious approach to avoiding ill-posed calibration problems where observation data is insufficient to constrain the parameters; a study of alternative choices was not undertaken, since methodologies in selecting the input parameter space are not the focus of this work. The spatial distribution of the selected sediment groups is indicated in Fig. 1.

Since the Bristol Channel and Severn Estuary contain significant intertidal regions, we include wetting and drying within Thetis using the scheme of Kärnä et al. (2011), where a modification is applied dynamically to the bathymetry in order to avoid negative water depth. This scheme is adopted since its formulation involves only a modification to the underlying equations; it therefore poses little additional complexity in differentiation of the model using numerical adjoint methods. This scheme introduces an additional wettingdrying parameter $\alpha$, which controls the transition from wet to dry regions, and is user-defined. In all Thetis simulations presented herein, $\alpha$ is taken to be $0.5 \mathrm{~m}$.

The mesh used for the simulations here is shown in Fig. 1, and was generated on a UTM30 coordinate projection, using the Python package qmesh (version 1.0.1) (Avdis et al., 2018), which interfaces the mesh generator Gmsh (version 2.10.1) (Geuzaine and Remacle, 2009). The mesh resolution varies from $1 \mathrm{~km}$ at the coastlines to $10 \mathrm{~km}$ in open regions, and the mesh contains a total of 8,704 triangular elements. Thetis is run using a $\mathrm{P}_{1}^{\mathrm{DG}}-\mathrm{P}_{1}^{\mathrm{DG}}$ discretisation, with a Crank-Nicolson timestepping scheme with a timestep $\Delta t=100 \mathrm{~s}$. Tidal dynamics are introduced through a Dirichlet boundary condition for the surface elevation $\eta$ at the ocean boundary, based on the eight leading constituents from the TPXO database (M2, S2, N2, K2, Q1, O1, P1, K1) (Egbert and Erofeeva, 2002). The bathymetry is subsampled from $30 \mathrm{~m}$ resolution data available from Digimap (Digimap, 2016).

\subsection{Thetis adjoint}

The adjoint method can be approached numerically either by discretising the analytically-derived adjoint equations (Funke et al., 2016), or by an algorithmic differentiation (AD) approach. In the conventional $\mathrm{AD}$ approach, adjoint source code is generated from the source code of the forward numerical model by a decomposition into elementary functions whose Jacobians can be easily computed (Bischof et al., 1992, Giering and Kaminski, 1998, Tber et al., 2007, Wilson et al., 2013). While "algorithmic", a perceived problem with this approach is that "a substantial amount of manual intervention is still necessary" (Sraj et al., 2014a), particularly in the maintenance of the adjoint code for every development in the forward model. In this work, the adjoint mode of Thetis is derived algorithmically via the use of the pyadjoint package (Mitusch et al., 2019). Pyadjoint takes an alternative AD approach, whereby the adjoint equations are derived at an abstracted level; this is facilitated by the abstracted approach of the Firedrake code generation framework underpinning Thetis (Rathgeber et al., 2016). The method therefore requires minimal 

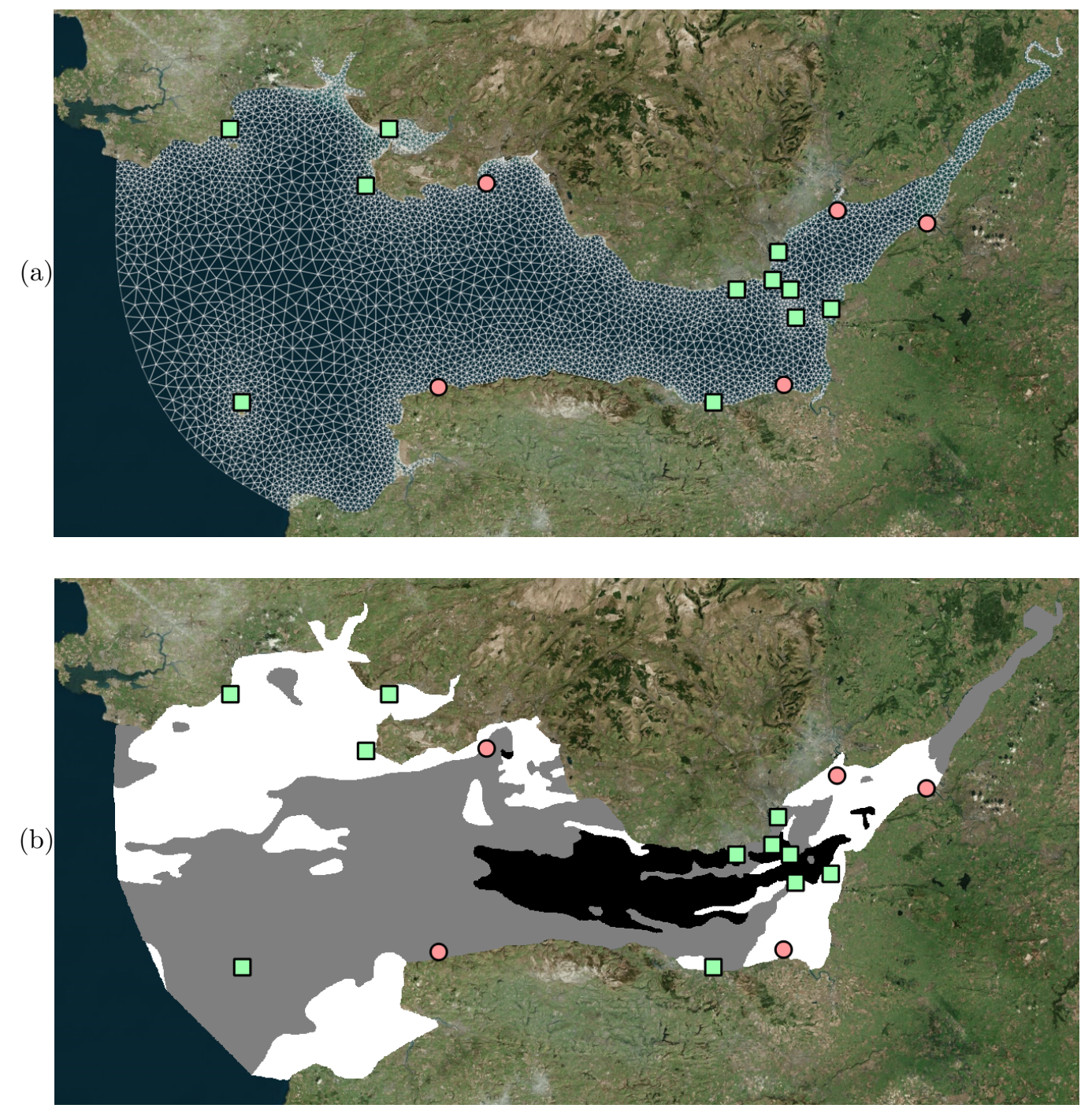

Figure 1: (a): Mesh used for all simulations within this paper. (b): Sediment groups; rock (black), gravel (grey), sand (white). Locations where tidal harmonic data are available are shown in both figures as green squares. BODC tide gauges are shown as red circles. 
manual intervention, and since the adjoint equations are solved within Firedrake in a similar manner to the forward equations, this results in an efficient implementation of the adjoint model.

In a general numerical modelling context, adjoint methods are used to compute the gradient of a model output (or functional), denoted $J$, with respect to a number of model inputs. In this work, the functional $J$ is chosen to be some measure of the misfit between the model and a set of observation data, and the inputs are the vector of unknown Manning coefficients $\mathbf{n}=\left(n_{1}, n_{2}, n_{3}\right)^{T}$. We then use the adjoint-computed gradient $\frac{\partial J}{\partial \mathbf{n}}$ within a gradient-based optimisation algorithm to find the set of Manning coefficients which minimises the misfit $J$. The power of the adjoint method lies in the fact that this gradient can be computed at a computational cost comparable to that of the forward model, independent of the number of parameters.

For further information on the use and implementation of algorithmic differentiation and the adjoint method, the reader is referred to Marotzke et al. (1999), Funke (2012), Mitusch et al. (2019) and references therein.

\subsection{Telemac}

In addition to Thetis, we also use the TELEMAC-2D model (Hervouet, 2007), henceforth referred to as Telemac. Telemac solves the shallow water equations in a similar form to Eq. (1), and is used here in its finite element mode with default continuous Galerkin discretisation, consisting of linear elements in velocity and depth; see the Telemac user manual for further detail (Lang et al., 2014). We use the same unstructured mesh as for the Thetis simulations, and a semi-implicit timestepping scheme with a timestep of $10 \mathrm{~s}$. The bottom friction formulation used for the Telemac simulations is identical to that used in Thetis, and Telemac results are used in later sections to validate the friction parameters selected by the Thetis-based calibration techniques.

\section{Calibration methods}

Here we describe the algorithms used for model calibration. The first method takes a Bayesian approach, determining the optimal parameters via a Markov Chain Monte Carlo method applied to the assimilation of tidal harmonic data. The second uses Thetis's adjoint model within a gradient-based optimisation algorithm, applied separately to tidal harmonic and timeseries tide gauge data.

\subsection{Assimilation of harmonic data using Bayesian inference}

Bayesian inference is a powerful statistical technique for inverse problems, and has been applied to bottom friction parameter estimation previously (Hall et al., 2011, Sraj et al., 2014b). Here we will use a Markov Chain Monte Carlo (MCMC) method, which relies on large numbers of model runs, and we therefore use a Gaussian process emulator as a computationally efficient surrogate model in place of the full Thetis model. This method has the advantage that the main computational cost is in the creation of the emulator training dataset, as the subsequent emulator training and MCMC algorithm can be performed at relatively low computational cost. 


\subsubsection{Gaussian process emulator}

A Gaussian process emulator (GPE) is a statistical model commonly used as a surrogate for computationally expensive numerical models. Here we use the Python package GPy (GPy, since 2012) to generate a Gaussian process emulator, which is used in place of the full Thetis model in the Bayesian inversion algorithm. Here we briefly describe Gaussian process emulation; for further detail the reader is referred to the GPy documentation (GPy, since 2012), and examples of the use of GPEs with MCMC methods, e.g. Hall et al. (2011), Tagade et al. (2013).

We employ a GPE to predict the full Thetis model outputs in the form of tidal harmonic amplitudes at the 11 locations indicated in Fig. 1; for a given constituent $C$, we denote the vector (of length 11) of modelled harmonic amplitudes by $\mathbf{G}_{C}(\mathbf{n})$ for a set of input parameters $\mathbf{n}$. Given a set of training data, the GPE gives the best linear unbiased prediction of full model outputs for unseen values of the model inputs. The GPE also exports a covariance matrix encapsulating the uncertainty in the GPE estimate. The GPE output interpolates the training data, with zero covariance at training points.

The training data is generated from a harmonic analysis (at the 11 locations) of Thetis model outputs. The Thetis training runs were configured using 40 samples from the input parameter space, drawn from uninformative priors in the range $[0.01,0.05]$, using Latin hypercube sampling. The parameter ranges were chosen based on typical base values for Manning coefficients from Arcement and Schneider (1989). According to the ' $10 d$ ' rule (Sobol, 2001, Hristov et al., 2017), it is common to train a Gaussian process emulator using at least $10 d$ samples, where $d$ is the number of input parameters; here we have three input parameters, and train using 40 samples.

We perform Bayesian inversion using two different sets of data. First we assimilate only M2 amplitude data, so that a direct comparison can be made with the adjoint gradient-based approach described in Section 3.2.1, which is applied to M2 amplitude data only. Secondly, in order to observe the effect of an increased volume of assimilated data on the estimated parameters and their uncertainty, we assimilate harmonic amplitude data for four semi-diurnal constituents (M2, S2, N2, K2). An initial 10-day spin-up is run using a uniform Manning coefficient $n=0.02 \mathrm{~s} \mathrm{~m}^{-1 / 3}$. Each training sample follows from this spin-up and is run for 30 days, with a full harmonic analysis performed at the 11 gauge locations, so that either combination of harmonics can be assimilated using the same emulator training dataset. We note that assimilation of M2 data could have been achieved using an emulator trained using a set of shorter model runs, but since the longer runs are required for the assimilation of multiple constituents, they are reused for the M2-only assimilation to save computational cost.

\subsubsection{Markov Chain Monte Carlo algorithm}

The Bayesian inversion framework follows a similar approach to Sraj et al. (2014b). We denote the set of observed tidal harmonic amplitudes $\left\{\mathbf{y}_{C}\right\}$, corresponding to the set of harmonic constituents $\{C\}=$ $\left\{C_{1}, C_{2}, \ldots\right\}$, where each $\mathbf{y}_{C}$ is a vector of length 11 (for the 11 tidal harmonic observation locations indicated 
in Fig. 1). The set of emulator outputs, estimated for a vector of Manning coefficients $\mathbf{n}=\left(n_{1}, n_{2}, n_{3}\right)^{T}$, is denoted $\left\{\mathbf{G}_{C}(\mathbf{n})\right\}$. Bayes' theorem gives

$$
\Pi\left(\mathbf{n} \mid\left\{\mathbf{y}_{C}\right\}\right) \propto L\left(\left\{\mathbf{y}_{C}\right\} \mid \mathbf{n}\right) \prod_{i=1}^{3} q\left(n_{i}\right)
$$

where $\Pi$ is the posterior distribution of the parameters $\mathbf{n}$ given the observed data $\left\{\mathbf{y}_{C}\right\}, L$ is the likelihood of observing the outputs $\left\{\mathbf{y}_{C}\right\}$ given the parameters $\mathbf{n}$, and $q$ is the prior distribution of each of the parameters $n_{i}$, which we take to be an uninformative prior in the range $[0.01,0.05]$ and hence

$$
q\left(n_{i}\right)= \begin{cases}\frac{1}{0.05-0.01} & \text { for } 0.01<n_{i}<0.05 \\ 0 & \text { otherwise. }\end{cases}
$$

For a constituent $C$, we assume that the model-observation discrepancies, which are the components of the vector $\mathbf{y}_{C}-\mathbf{G}_{C}(\mathbf{n})$, are independent and identically distributed variables with zero mean and variance $\sigma_{C}^{2}$. The likelihood $L\left(\left\{\mathbf{y}_{C}\right\} \mid \mathbf{n}\right)$ for a set of constituents $C$ is therefore given by

$$
L\left(\left\{\mathbf{y}_{C}\right\} \mid \mathbf{n}\right)=\prod_{C}\left[\left(2 \pi \sigma_{C}^{2}\right)^{-N / 2} \exp \left(-\frac{1}{2} \frac{\left|\mathbf{y}_{C}-\mathbf{G}_{C}(\mathbf{n})\right|^{2}}{\sigma_{C}^{2}}\right)\right]
$$

The covariance in the model outputs $\mathbf{G}_{C}(\mathbf{n})$ due to the use of the GPE, which is estimated as part of the GPE evaluation, is assumed to be negligible compared to the variances $\sigma_{C}^{2}$, and neglected within the Bayesian inversion. Since these $\sigma_{C}^{2}$ are unknown a priori, they are treated as hyperparameters, i.e. they are included as additional parameters to be inferred by the MCMC algorithm. We denote the full vector of unknowns $\boldsymbol{\theta}=\left(n_{1}, n_{2}, n_{3}, \log \sigma_{C_{1}}^{2}, \log \sigma_{C_{2}}^{2}, \ldots\right)$, and the full posterior distribution is therefore given by

$$
\Pi\left(\boldsymbol{\theta} \mid\left\{\mathbf{y}_{C}\right\}\right) \propto \prod_{C}\left[\left(2 \pi \sigma_{C}^{2}\right)^{-N / 2} \exp \left(-\frac{1}{2} \frac{\left|\mathbf{y}_{C}-\mathbf{G}_{C}(\mathbf{n})\right|^{2}}{\sigma_{C}^{2}}\right)\right] \prod_{i=1}^{3} q\left(n_{i}\right) \prod_{C} q\left(\sigma_{C}^{2}\right) .
$$

For the unknown variances $\sigma_{C}^{2}$, the only prior information is that they must be positive. We therefore follow Sraj et al. (2014b) and assume Jeffreys priors (Sivia and Skilling, 2006), such that

$$
q\left(\sigma_{C}^{2}\right)= \begin{cases}\frac{1}{\sigma_{C}^{2}} & \text { for } \sigma_{C}^{2}>0 \\ 0 & \text { otherwise. }\end{cases}
$$

The posterior distribution $\Pi\left(\boldsymbol{\theta} \mid\left\{\mathbf{y}_{C}\right\}\right)$ of Eq. (6) gives the probability distribution of the unknown Manning coefficients and variances $\sigma_{C}^{2}$, given the set of observations $\left\{\mathbf{y}_{C}\right\}$, and its evaluation represents the model calibration problem. A technique for sampling this posterior distribution when it cannot be directly calculated is the Markov Chain Monte Carlo (MCMC) method, which has the advantage that the constant of proportionality need not be determined. We use an implementation of the Random Walk Metropolis Hastings MCMC algorithm (Hastings, 1970), which is given by Algorithm 1. The algorithm requires the selection of an appropriate proposal distribution covariance matrix, $\Sigma_{\text {step }}$, governing the size of the random 
steps within the parameter space. We set

$$
\Sigma_{\text {step }}=\left[\begin{array}{cccccc}
0.001^{2} & 0 & 0 & 0 & \ldots & 0 \\
0 & 0.001^{2} & 0 & 0 & \ldots & 0 \\
0 & 0 & 0.001^{2} & 0 & \ldots & 0 \\
0 & 0 & 0 & 0.1^{2} & \ldots & 0 \\
\vdots & \vdots & \vdots & \vdots & \ddots & \vdots \\
0 & 0 & 0 & 0 & \ldots & 0.1^{2}
\end{array}\right]
$$

so that the random steps in each of the Manning coefficients have zero mean and a standard deviation of $0.001 \mathrm{~s} \mathrm{~m}^{-1 / 3}$, and the random steps in each $\log \sigma_{C}^{2}$ have zero mean and a standard deviation of 0.1 . These step sizes were found to give satisfactory results, without the need for an adaptive MCMC algorithm.

In the results presented here, we take $M=10^{6}$ samples, discarding the first $2 \cdot 10^{5}$ as a burn-in period, and the resulting chain of values $\boldsymbol{n}^{[i]}$ generated by the MCMC algorithm constitute samples from the posterior distribution. We can therefore visualise the joint probability distribution of the input parameters given the set of observations by a histogram of these $\boldsymbol{n}^{[i]}$ values, which we smooth through kernel density estimation. The mean and standard deviation of the samples correspond to a best estimate, and uncertainty, of each parameter.

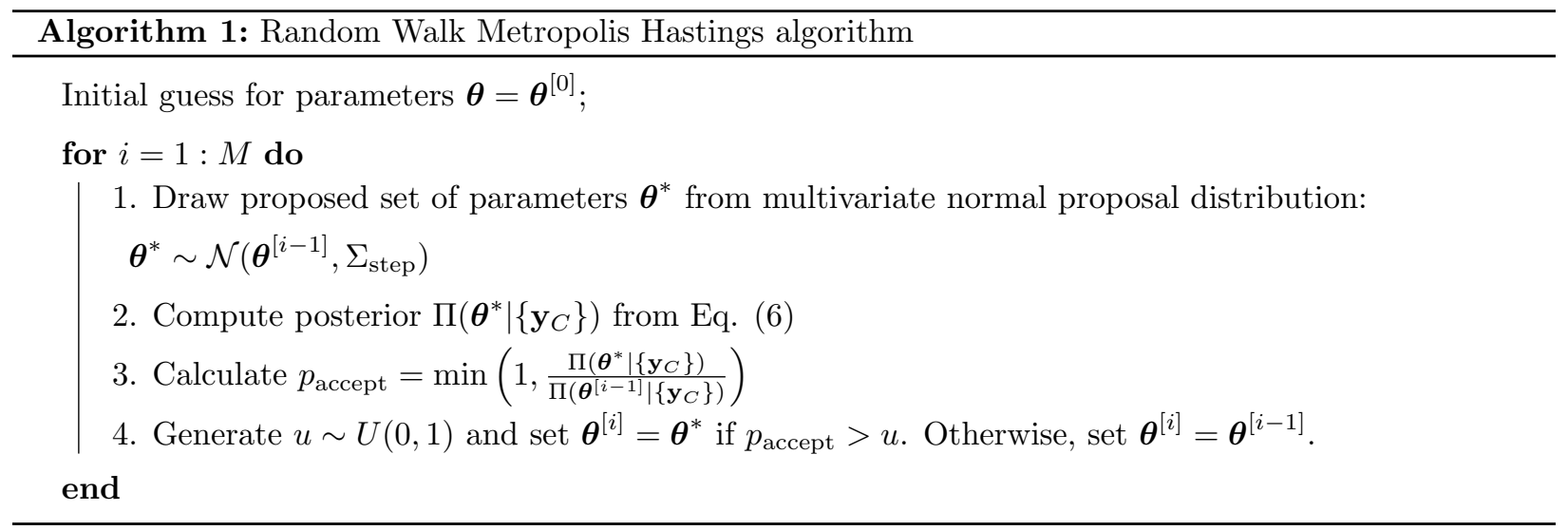

\subsection{Gradient-based optimisation using Thetis adjoint}

The use of adjoint methods facilitates the solution of the model calibration problem via gradient-based optimisation. We define a functional $J$, which measures some misfit between model and observations, which we seek to minimise. This minimisation is performed using the $L$-BFGS-B gradient-based optimisation algorithm (Zhu et al., 1997) within the Python scientific computing library SciPy. Each iteration of the algorithm requires a forward and adjoint run of Thetis, in order to calculate the functional value $J$ and its gradient vector $\frac{\partial J}{\partial \mathbf{n}}$, respectively. The algorithm is terminated when the change in the functional value between successive iterations falls below $10^{-6}$ of the original functional value, i.e. when $|\Delta J|_{i}<10^{-6} \cdot J_{0}$, 
where the subscript denotes the iteration number. Here we make two choices for the misfit functional $J$, based on the two sources of data described in Section 2.1.

\subsubsection{Assimilation of harmonic data}

Here we seek to assimilate tidal harmonic constituent data from the 11 gauges indicated in Fig. 1. The challenge in assimilating this data via a numerical adjoint model is the high demand on placed on computational resources, in particular memory, by adjoint models. This is compounded by the definition of the misfit between the model and harmonic tide gauge data, which requires a sufficiently long forward run that a harmonic analysis may be performed on the simulated timeseries. In order to limit the computational cost of assimilating harmonic data, we opt to assimilate only the M2 harmonic, so that a short assimilation period of 3 days is sufficient. For this, the model is forced with the M2 tidal constituent only, and after an initial spin-up period of 10 days with a uniform Manning coefficient $n=0.02 \mathrm{~s} \mathrm{~m}^{-1 / 3}$, the forward and adjoint models are run for 3.5 days for each iteration. Out of the 3.5 day simulation period, the first 12 hours are used as additional spin-up with the updated friction parameters, to eliminate the dependence of the model outputs on the spin-up parameters. After performing a harmonic analysis (for the M2 constituent only) based on the final 3 days of each simulation, the model-observation misfit is defined as

$$
J=\sum_{i=1}^{11}\left(A_{M, i}-A_{O, i}\right)^{2},
$$

where $A_{M, i}$ and $A_{O, i}$ are the modelled and observed M2 amplitudes at the 11 gauges $i$, respectively. Note that the minimisation of this misfit is equivalent to the maximisation of the posterior of Eq. (6) for a single constituent, i.e. this method constitutes maximum likelihood estimation. We therefore expect results from this calibration method to be consistent with those from the MCMC method applied to the M2 constituent.

The advantage of this method over the MCMC approach is its use of an efficient gradient-based algorithm via the use of the adjoint model, without needing to approximate the numerical model with a surrogate. However, the computational resources available for this work prevent a thorough harmonic analysis based on more than one tidal constituent.

\subsubsection{Assimilation of timeseries data}

There are five locations within the model domain at which free surface elevation timeseries are sampled at 15 minute intervals (see red circles in Fig. 1). To assimilate this data, model-observation misfit is computed over a 24-hour period commencing on $21^{\text {st }}$ June 2010. This assimilation period was chosen to coincide with relatively calm meteorological conditions, since we seek parameters calibrated for tide-only performance and for simplicity do not include any meteorological effects in the model. The model is first run for a spin-up time of 10 days prior to the start of the assimilation period, with a uniform Manning coefficient $n=0.02$ $\mathrm{s} \mathrm{m}^{-1 / 3}$. Each optimisation iteration commences with an additional 12-hour spin-up with the updated set of Manning coefficients, before the 24-hour assimilation period. 
For comparisons between the model and this timeseries data, the misfit functional is defined as

$$
J=\sum_{i=1}^{5} \int_{T_{0}}^{T_{1}}\left(\eta_{M i}(t)-\eta_{O i}(t)\right)^{2} \mathrm{~d} t
$$

where $\eta_{M i}$ and $\eta_{O i}$ are the modelled and observed surface elevations at each of the five tide gauges $i$, respectively, and the time integral spans the 24-hour period described above and is approximated by a suitable discrete method applied to the 15 minute data intervals.

This method has the advantage that each optimisation iteration requires forward and adjoint model runs of only 36 hours each (12-hour spin-up plus 24-hour assimilation window), and the computational cost of the optimisation algorithm is therefore relatively low. However, tide gauge timeseries data are available at only five locations within the model domain, all confined to be close to the coastline, and the assimilated observations must all have been obtained during the same time period to keep the assimilation window short. The assimilation window must also coincide with calm meteorological conditions to avoid the need to model the effects of wind stress and atmospheric pressure gradient.

\section{Results}

\subsection{Synthetic experiment}

In order to validate the calibration methods, we first use a 'synthetic' experiment. Accordingly, the Thetis model is run for one month using specified friction parameters $\mathbf{n}=(0.036,0.027,0.022)^{T} \mathrm{~s} \mathrm{~m}^{-1 / 3}$. The resulting model outputs are used as 'observation' data for each calibration algorithm, to test that the calibration methods constitute well-posed problems, and are capable of recovering the synthetic friction parameters to acceptable accuracy and at a reasonable computational cost.

The results of this synthetic experiment are summarised in Tables 1 and 2. They show the values of the calibrated parameters for each method, and the number of forward and adjoint runs taken by each method to achieve the result, respectively. We comment on each of the calibration methods/datasets:

\section{MCMC assimilation of harmonic data, M2 only}

Since the true model-observation variance $\sigma_{C}^{2}$ for this synthetic case is zero, we have specified a fixed $\sigma_{C}^{2}=0.0025 \mathrm{~m}^{2}$ within the MCMC method; this is equivalent to assuming an uncertainty $\sigma_{C}=0.05 \mathrm{~m}$ in the synthetic observations. The resulting posterior joint probability density function (PDF) is shown in Fig. 2, and we observe that the 'true' values of the friction coefficients lie close to the peak in the posterior PDFs. There is strong covariance between estimated Manning coefficients corresponding to rock and gravel, while the coefficient for sand shows little covariance with the other parameters.

\section{MCMC assimilation of harmonic data, 4 constituents}

We again simulate uncertainty in the observations by specifying a fixed $\sigma_{C}^{2}=0.0025 \mathrm{~m}^{2}$ for all constituents $C$. The resulting joint PDF is shown in Fig. 3, and is very similar to the result from the assimilation of only the M2 constituent. In general, the resulting PDF is narrower, as reflected both 


\begin{tabular}{c|c|c|c} 
& $n_{1}$ & $n_{2}$ & $n_{3}$ \\
\hline True value & 0.036 & 0.027 & 0.022 \\
MCMC result, M2 only & $0.037 \pm 0.007$ & $0.027 \pm 0.009$ & $0.022 \pm 0.005$ \\
MCMC result, 4 constituents & $0.036 \pm 0.005$ & $0.028 \pm 0.007$ & $0.022 \pm 0.004$ \\
Harmonic adjoint calibration result & 0.0360 & 0.0270 & 0.0220 \\
Timeseries calibration result & 0.0359 & 0.0271 & 0.0220
\end{tabular}

Table 1: Summary of estimated Manning coefficients (units $\mathrm{sm}^{-1 / 3}$ ) for the 'synthetic' experiment. All calibration methods recover the synthetic friction values well.

\begin{tabular}{c|c|c|} 
& Forward runs & Adjoint runs \\
\hline MCMC result, M2 only & $40 \times 28$ days & \\
MCMC result, 4 constituents & $40 \times 28$ days & \\
Harmonic adjoint calibration result & $15 \times 3.5$ days & $15 \times 3.5$ days \\
Timeseries calibration result & $16 \times 1.5$ days & $16 \times 1.5$ days
\end{tabular}

Table 2: Summary of computational demand for the 'synthetic' experiment. The MCMC run-count is for the training data set; the computational cost of training and running the GPE is negligible compared to the full model.

in the histograms of Fig. 3 and the uncertainties in the estimated parameter values of Table 1. This shows that assimilation of additional data can be used to overcome uncertainty in observation data, resulting in a tighter constraint on the unknown parameters. However, the high covariance between the estimated rock and gravel friction parameters remains present.

\section{Adjoint-based assimilation of harmonic data}

The algorithm converged in 14 iterations, requiring a total of 15 model runs; this includes line search steps as part of the optimisation algorithm, hence this is greater than the number of iterations. The implementation of the L-BFGS-B optimisation algorithm performs equal numbers of forward and adjoint runs, even in line search steps. The misfit functional $J$ decreased to approximately $3 \cdot 10^{-7 \%}$ of its original value, with the synthetic Manning coefficients recovered to within an absolute value of $2 \cdot 10^{-5} \mathrm{~s} \mathrm{~m}^{-1 / 3}$.

\section{Adjoint-based assimilation of timeseries data}

The algorithm converged in 9 iterations, requiring a total of 16 forward and adjoint runs, where this again includes line search steps. The misfit functional $J$ was decreased to less than $3 \cdot 10^{-8 \%}$ of its original value, with the Manning coefficients converging to within an absolute value of $1.2 \cdot 10^{-6} \mathrm{~s} \mathrm{~m}^{-1 / 3}$ of the prescribed synthetic values. 

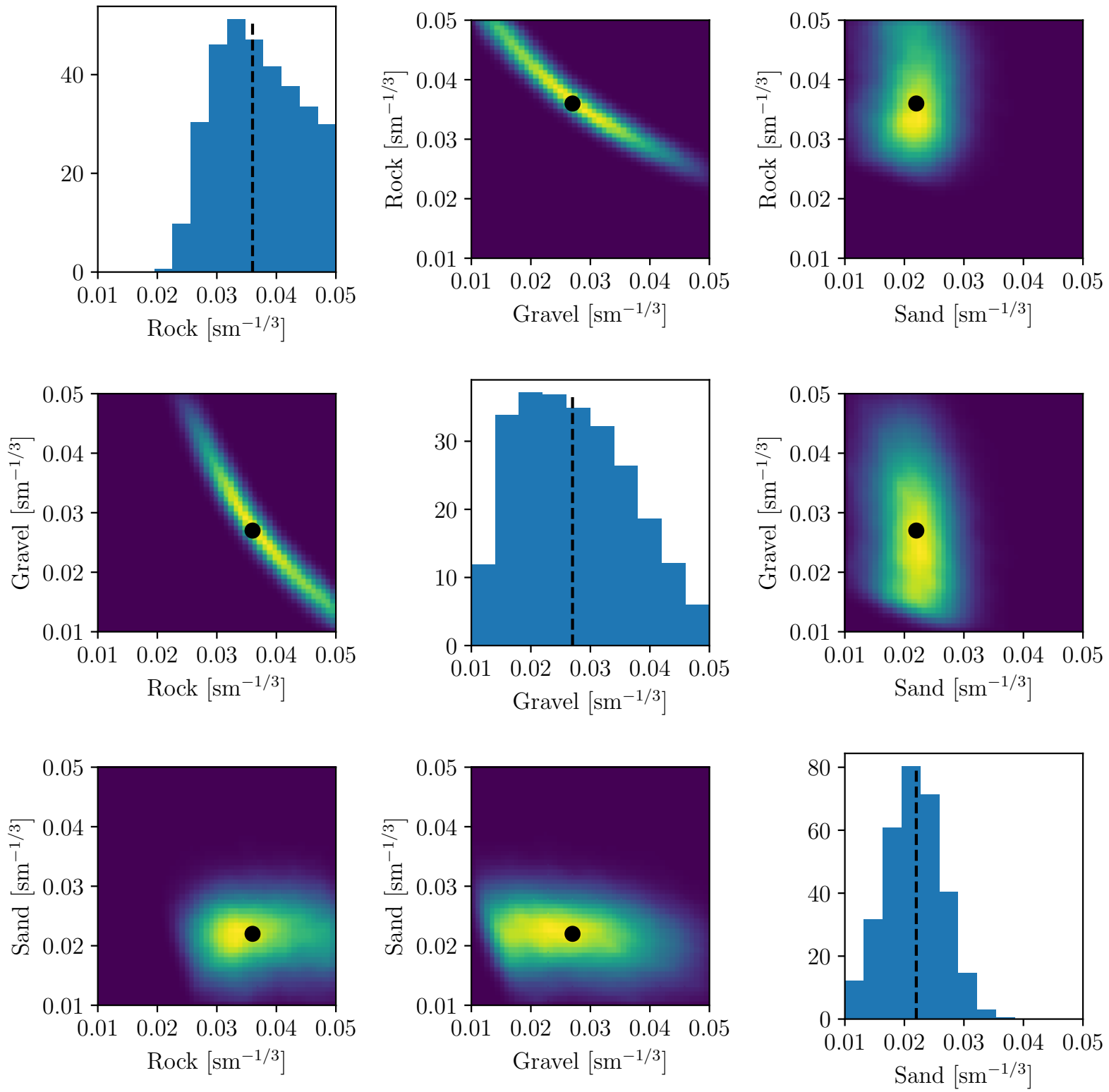

Figure 2: Posterior joint probability density function (PDF) obtained from MCMC sampling, based on assimilation of M2 harmonic amplitudes, using 'synthetic' observations and using a fixed $\sigma_{C}^{2}=0.0025 \mathrm{~m}^{2}$. Distributions are smoothed using kernel density estimation. Black points/lines show original synthetic friction values, and lie well within the MCMC-estimated PDF. 

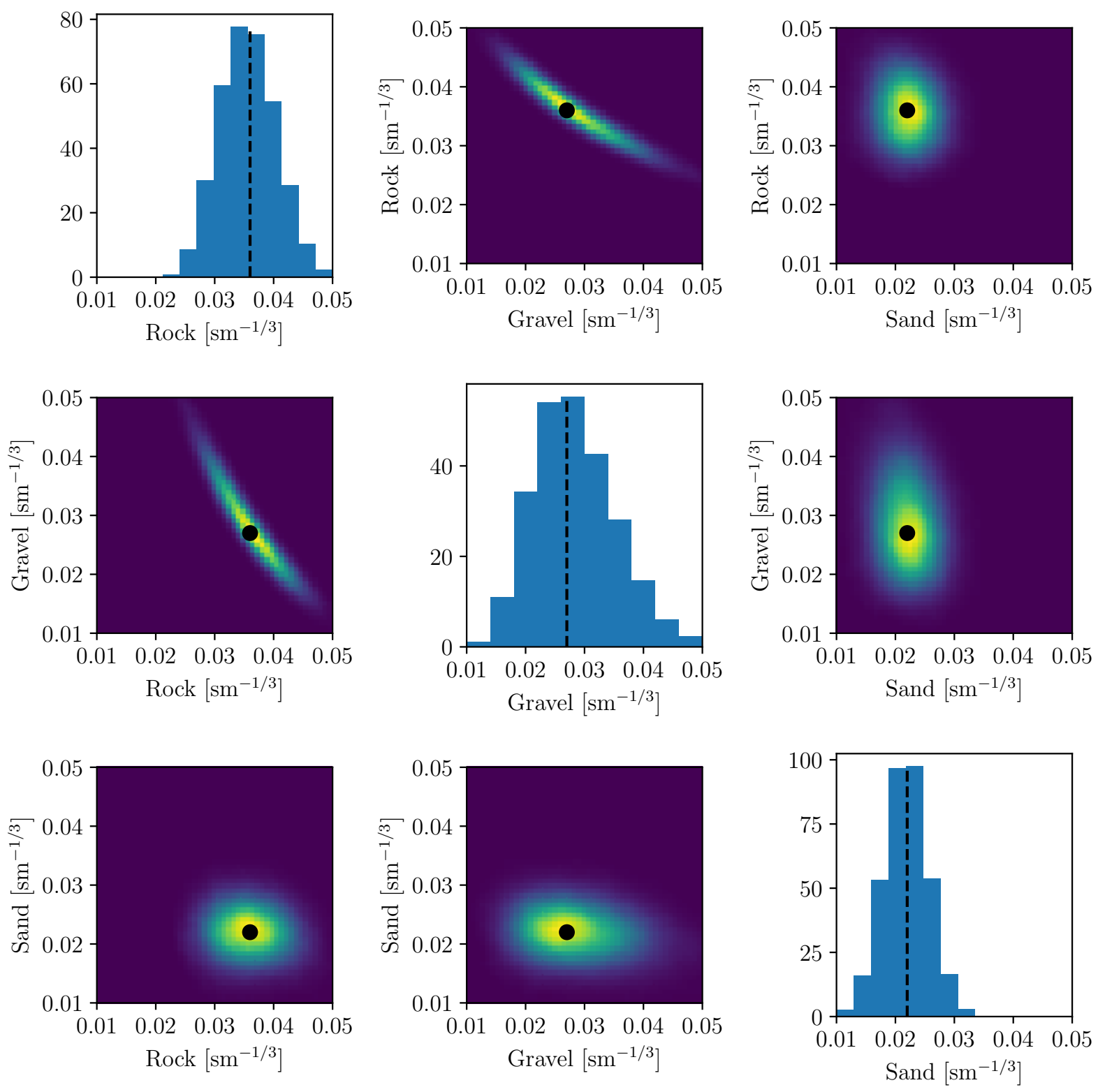

Figure 3: Posterior joint probability density function (PDF) obtained from MCMC sampling, based on assimilation of M2, $\mathrm{S} 2$, N2 and K2 harmonic amplitudes, using 'synthetic' observations and using a fixed $\sigma_{C}^{2}=0.0025 \mathrm{~m}^{2}$. Distributions are smoothed using kernel density estimation. Black points/lines show original synthetic friction values, and lie well within the MCMC-estimated PDF. The PDF exhibits reduced uncertainty (spread) compared with Fig. 2. 


\subsection{Assimilation of real data}

We now apply each calibration method to the assimilation of real data, with results summarised in Tables 3 and 4 . We observe the following:

\section{MCMC assimilation of harmonic data, M2 only}

The posterior joint PDF of the estimated friction parameters is shown in Fig. 4, along with a histogram of the $\log \sigma_{M 2}^{2}$ values. The estimated covariance matrix due to the GPE has elements on the order of $10^{-7}$ or smaller, and the magnitude of the inferred values for $\sigma_{M 2}^{2}$ therefore justifies our assumption that the GPE covariances can be neglected. Similarly to the corresponding 'synthetic' assimilation, there is strong covariance between the estimated coefficients for rock and gravel, while the coefficient for sand shows no significant covariance with the other parameters. The estimated sand coefficient lies at the lower bound of the prior distribution.

\section{MCMC assimilation of harmonic data, 4 constituents}

The posterior joint PDF is shown in Fig. 5, along with histograms of each of the $\log \sigma_{C}^{2}$ values. The estimated Manning coefficients are consistent with the M2-only MCMC result, but exhibit smaller uncertainties due to the additional data assimilated. In this case, the covariance between the coefficients for rock and gravel makes them almost indistinguishable, and we again find that the sand coefficient lies on the lower bound of the prior distribution.

\section{Adjoint-based assimilation of harmonic data}

The convergence of the L-BFGS-B algorithm is shown in Fig. 6. The misfit is reduced to approximately $2 \%$ of its original value during the optimisation, which converges after 15 iterations, using a total of 16 forward and adjoint runs. The resulting estimated friction parameters are consistent with the distributions estimated by both MCMC results.

\section{Adjoint-based assimilation of timeseries data}

The convergence for the assimilation of timeseries data is shown in Fig. 6. The misfit is reduced to around $25 \%$ of its original value, converging after 11 iterations, with a total of 12 forward and adjoint runs; this is a lower computational cost than for the equivalent synthetic experiment, although a relatively poor reduction in the misfit functional is achieved. The resulting Manning coefficients are somewhat different from those estimated by the assimilation of harmonic data (particularly the gravel coefficient), but still consistent with the uncertainty estimate of the M2-only MCMC approach.

There is no 'correct' set of calibrated friction parameters to directly validate the calibrated parameter sets returned by each calibration method. Instead, we apply each set of calibrated parameters to both Thetis and Telemac forward model runs, and identify three measures of the model-observation misfit, as follows:

(i) Normalised RMSE (NRMSE) of modelled timeseries, based on the square root of Eq. (10), computed using Thetis simulation results. This measure will reveal how successful the assimilation of harmonic data has been in improving the modelled timeseries. 


\begin{tabular}{c|c|c|c} 
& $n_{1}$ & $n_{2}$ & $n_{3}$ \\
\hline MCMC result, M2 only & $0.037 \pm 0.007$ & $0.029 \pm 0.009$ & $0.014 \pm 0.003$ \\
MCMC result, 4 constituents & $0.034 \pm 0.005$ & $0.033 \pm 0.007$ & $0.012 \pm 0.002$ \\
Harmonic adjoint calibration result & 0.0369 & 0.0302 & 0.0161 \\
Timeseries calibration result & 0.0336 & 0.0243 & 0.0155
\end{tabular}

Table 3: Summary of estimated Manning coefficients (units $\mathrm{s}^{-1 / 3}$ ) for the assimilation of real data.

(ii) NRMSE of eight harmonic amplitudes, equivalent to the square root of Eq. (9) but extended to the eight leading order constituents (M2, S2, N2, K2, Q1, O1, P1, K1), computed using Thetis. Note that this is a comparison using a greater number of harmonics than used by any of the calibration methods. This measure will indicate how successful the assimilation of timeseries data, or different combinations of harmonic constituent data, has been in improving overall harmonic-based error.

(iii) The same eight-harmonic NRMSE as in (ii) above, using results from Telemac. The use of a second numerical model will indicate how successful each calibration method has been in identifying non-model specific friction parameters.

The results for each of these misfit measures are summarised in Table 5. For comparison purposes we have also included optimal results using single-coefficient (spatially uniform) friction parameters for each misfit measure, which were determined from a brute force approach using model runs with values of $n$ from 0.01 to $0.05 \mathrm{~s} \mathrm{~m}^{-1 / 3}$ in steps of 0.0025 , and taking the minimum NRMSE. We make three observations:

(i) All four calibration experiments produce friction parameters which improve all three measures of modelobservation misfit, compared with the initial guess (a uniform Manning coefficient of $0.02 \mathrm{~s} \mathrm{~m}^{-1 / 3}$ ).

(ii) The minimum achievable misfit to a given set of observation data is reduced by the use of a sedimentbased friction coefficient, compared with an optimally-selected spatially uniform coefficient. For example, the Thetis timeseries NRMSE, using the sediment-based parameters selected by the calibration method which directly assimilated timeseries data, is $7.2 \%$, In contrast, the best result achieved using a uniform coefficient is $8.4 \%$. This shows the reduction in misfit which can be achieved by increasing the number of degrees of freedom in the tuning parameters.

(iii) For Telemac, the use of the friction parameters selected using either the adjoint harmonic calibration or M2-only MCMC calibration results in a smaller NRMSE than can be achieved using any uniform coefficient, despite the sediment-based optimal values being selected using a different model (Thetis).

Taking into account all three NRMSE values for each calibration method, we suggest that the M2-only MCMC method produces the best estimate of the unknown friction parameters. 

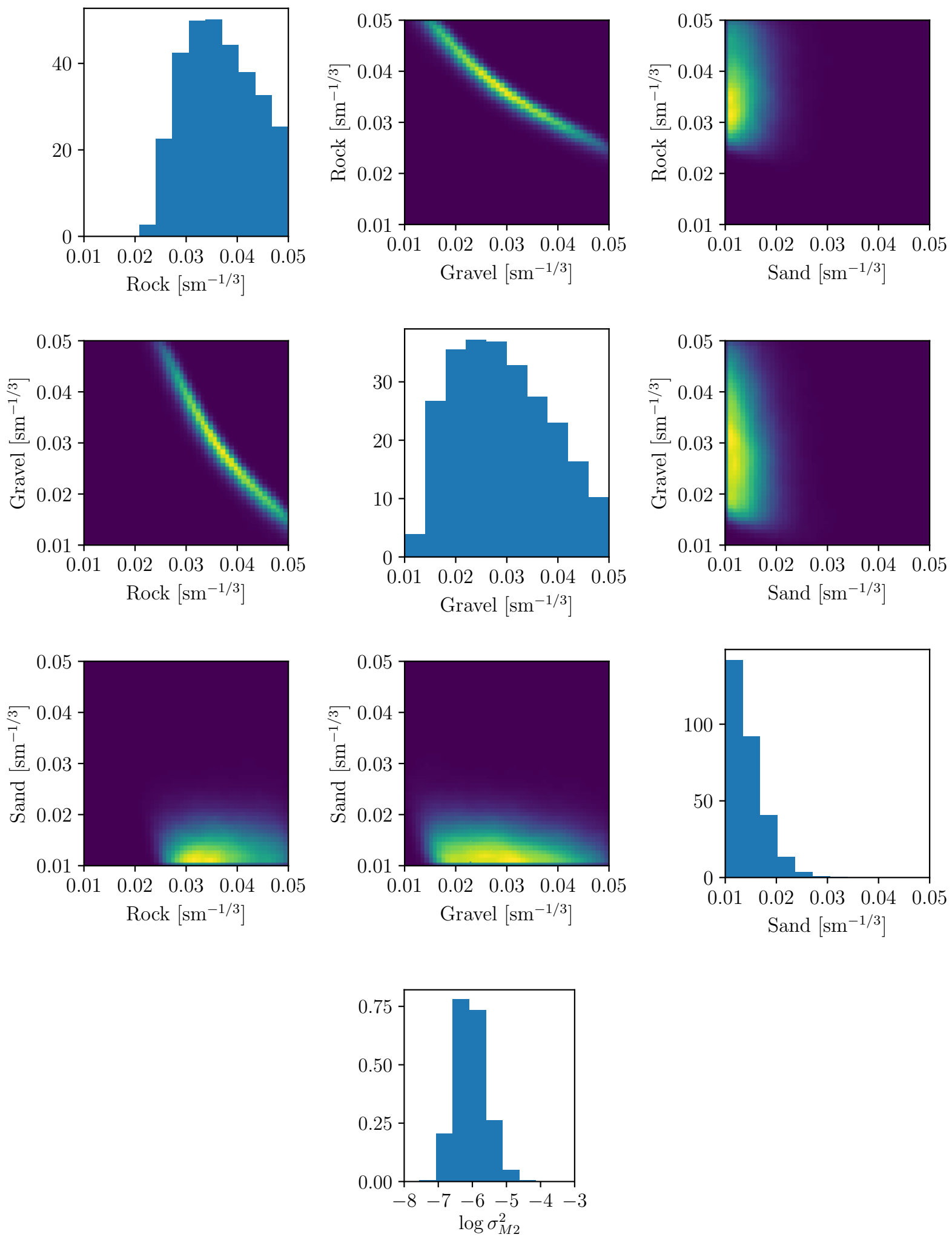

Figure 4: Posterior probability density function obtained from MCMC sampling, based on assimilation of M2 harmonic amplitudes, using real observations. Distributions are smoothed using kernel density estimation. 

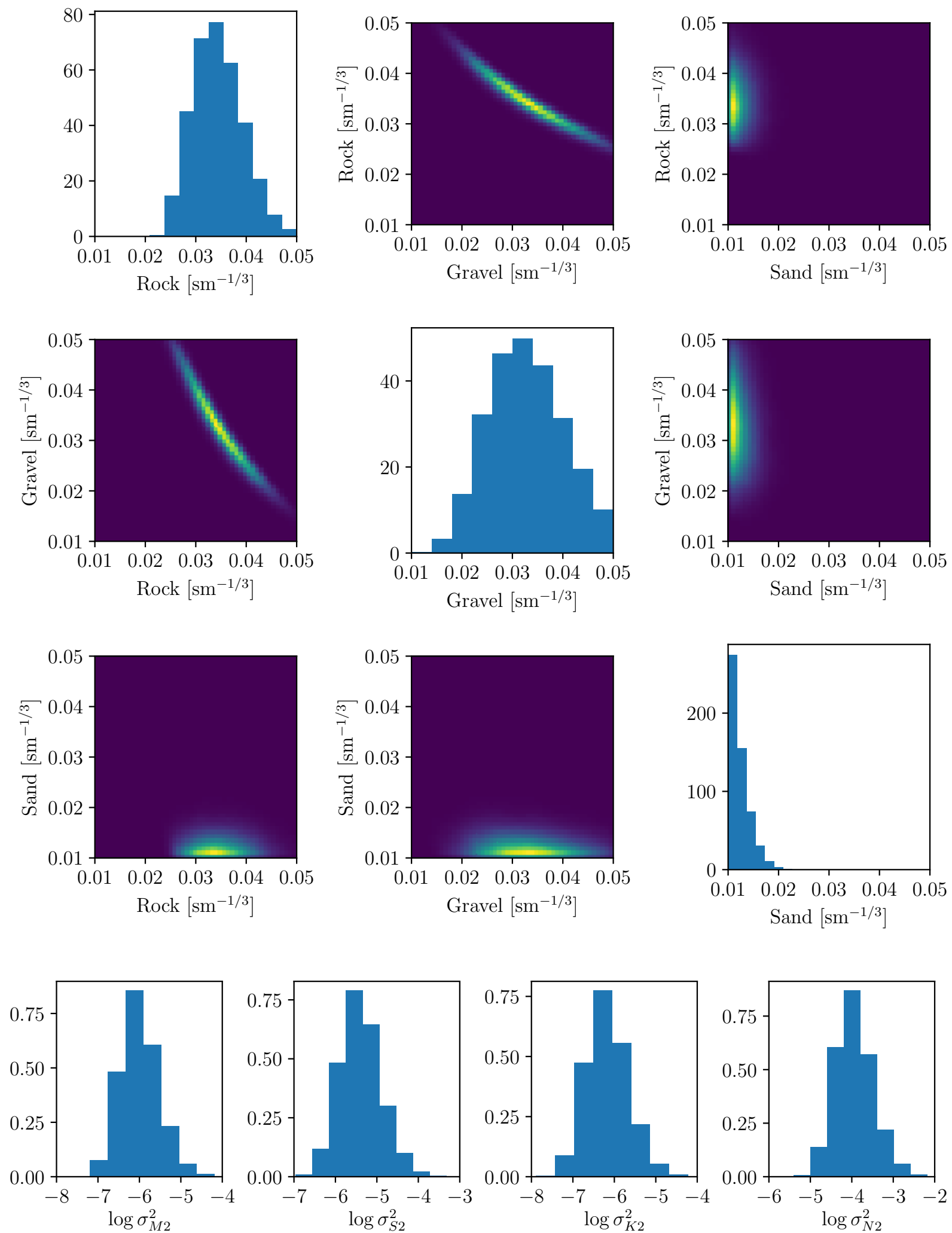

Figure 5: Posterior probability density function obtained from MCMC sampling, based on assimilation of M2, S2, K2 and N2 harmonic amplitudes, using real observations. Distributions are smoothed using kernel density estimation. 

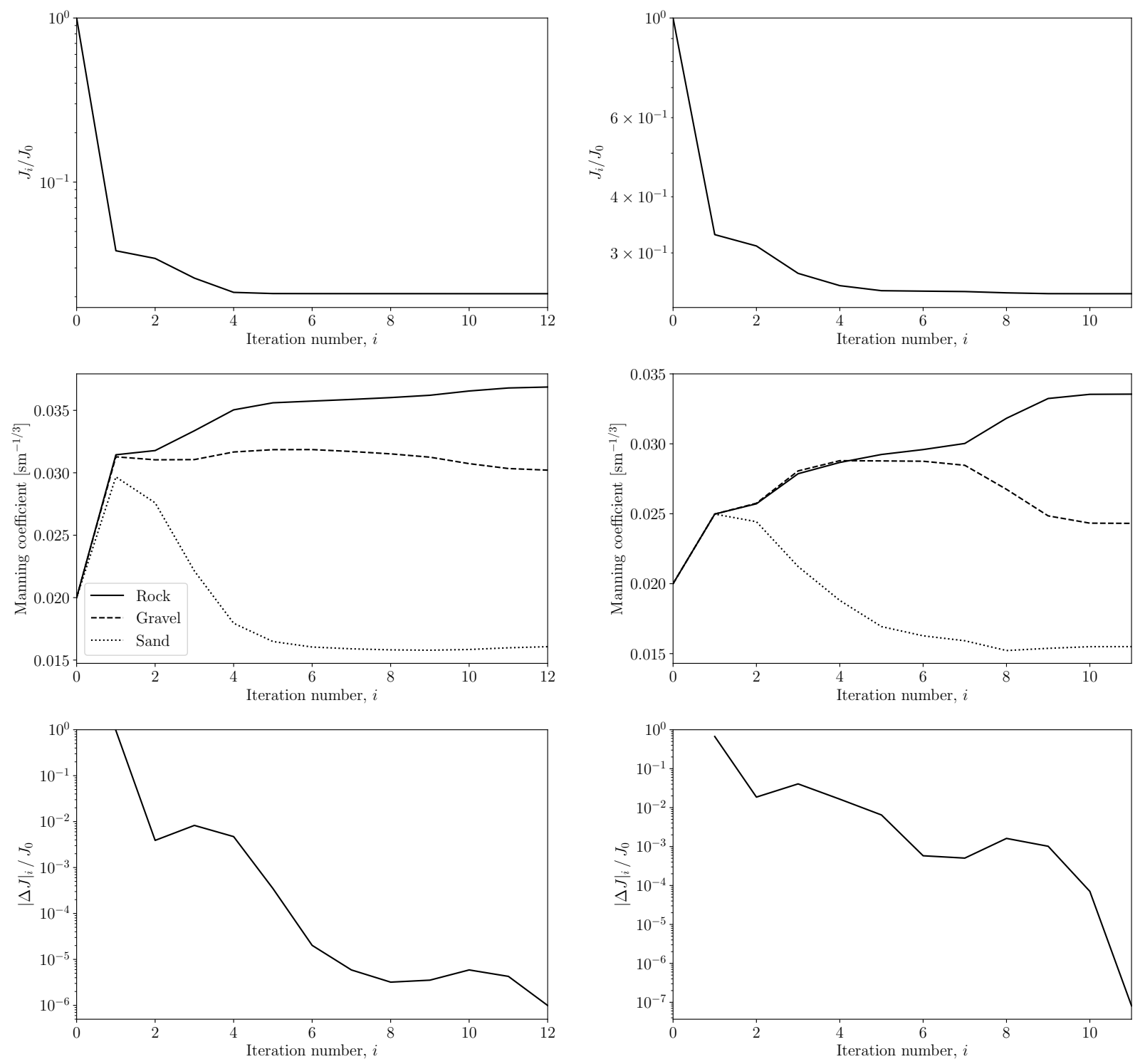

Figure 6: Results from adjoint-based assimilation of M2 harmonic data (left) and timeseries data (right) using L-BFGS-B algorithm. Top: Evolution of misfit functional. Middle: Evolution of estimated parameter values. Bottom: Convergence.

\begin{tabular}{c|c|c} 
& Forward runs & Adjoint runs \\
\hline MCMC result, M2 only & $40 \times 28$ days & \\
MCMC result, 4 constituents & $40 \times 28$ days & \\
Harmonic adjoint calibration result & $16 \times 3.5$ days & $16 \times 3.5$ days \\
Timeseries calibration result & $12 \times 1.5$ days & $12 \times 1.5$ days
\end{tabular}

Table 4: Summary of computational demand of each calibration method, for the assimilation of real data. 


\begin{tabular}{c|c|c|c} 
Calibrated parameters & Thetis timeseries & Thetis harmonic & Telemac harmonic \\
\hline Initial guess $\left(n=0.02 \mathrm{~s} \mathrm{~m}^{-1 / 3}\right)$ & $13.9 \%$ & $9.9 \%$ & $9.7 \%$ \\
MCMC, M2 only & $\mathbf{9 . 2 \%}$ & $\mathbf{4 . 5 \%}$ & $\mathbf{5 . 5 \%}$ \\
MCMC, 4 constituents & $9.2 \%$ & $4.4 \%$ & $6.0 \%$ \\
Adjoint harmonic calibration & $10.6 \%$ & $4.6 \%$ & $5.5 \%$ \\
Adjoint timeseries calibration & $7.2 \%$ & $5.4 \%$ & $6.2 \%$ \\
\hline Uniform $n=0.025 \mathrm{~s} \mathrm{~m}^{-1 / 3}$ & $8.4 \%$ & & \\
Uniform $n=0.03 \mathrm{~s} \mathrm{~m}^{-1 / 3}$ & & $5.1 \%$ & \\
Uniform $n=0.0275 \mathrm{~s} \mathrm{~m}^{-1 / 3}$ & & & $5.9 \%$
\end{tabular}

Table 5: Summary of misfit measures using calibration results. Values are normalised root mean squared errors as described in Section 4.2. Coefficients of $0.025,0.03$ and $0.0275 \mathrm{~s} \mathrm{~m}^{-1 / 3}$ are the optimal uniform-coefficient values for the Thetis timeseries, Thetis harmonic and Telemac harmonic NRMSEs, respectively, and are included for comparison purposes. We suggest that the M2-only MCMC result (in bold) produces the best overall result, and therefore the best estimate of the unknown Manning coefficients.

\section{Discussion}

We have shown in Section 4 that all of the calibration methods perform well both in the synthetic experiment, where the prescribed friction coefficients are accurately recovered by all methods, and in the assimilation of real data, where all estimated friction parameter sets are consistent (within the uncertainties estimated by the M2-only MCMC method). For the assimilation of real data, all four calibration experiments produce parameters which offer an improvement across all three measures of misfit, compared with the initial guess for the parameters. This includes the misfit as measured using a second model, Telemac. Furthermore, we find that the calibrated parameter sets selected by the harmonic adjoint-based or M2-only MCMC methods produce lower Telemac NRMSE values than any uniform friction coefficient (i.e. not only compared to the naive initial guess), and that the results from the other calibration methods also come very close to this optimal uniform-coefficient performance. This suggests that the calibration methods have avoided excessive influence from model-specific errors, and that the selection of the input parameter space (i.e. the division into three sediment groups) was appropriate to the observation data available. As a result, the calibration methods converge to physically meaningful parameters which are therefore applicable across different numerical models. It should be noted that the two numerical models used here employ similar equations and have used the same mesh, although the details of the finite element discretisation are different. Future work would be needed to investigate the applicability of calibrated friction parameters across models which differ more significantly.

In the PDFs of Figs 4 and 5, we observe that the Manning coefficient for sand is estimated to be at the lower bound of the prior distribution. This highlights the need for a physically motivated prior distribution; we found through additional numerical experiments that the reduction of this lower bound 
resulted in parameter sets which produced greater NRMSEs (computed using eight harmonics as described in Section 4.2), and therefore that the placement of the lower bound was influential. We also observe that the posterior PDFs exhibit strong covariance between the gravel and rock friction coefficients, indicating that the assimilated data are not sufficient to distinguish between the two parameters. This suggests that a similar result could have been achieved by combining the rock and gravel sediment groups together and performing a two-parameter calibration instead. Data at additional locations may be helpful in distinguishing these two parameters, and a sensitivity analysis-based framework such as that of Graham et al. (2017) could be utilised to propose new observation locations to better constrain the unknown friction parameters, but is beyond the scope of this work.

In addition to comparing the performance of the calibrated parameter sets, we can also compare the computational cost of each calibration method, as outlined in tables 2 and 4 . Here it important to consider the relative computational cost of forward and adjoint model runs, since the computational cost of a wellimplemented adjoint model is typically greater than that of the forward model by a multiplier slightly larger than unity (Griewank and Walther, 2008). For the Thetis adjoint model, we find that this multiplier is approximately 3.6. The gradient-based calibration using timeseries data is therefore the least computationally expensive of the methods used within this work, due to its short 1.5-day runs, while the MCMC approach is the most expensive, due to the long 28-day runs used to train the surrogate model. However, note that the same training dataset was used to train emulators for both the MCMC-based assimilation of data for four semi-diurnal constituents, and the MCMC-based assimilation of only the M2 harmonic amplitude; the choice of a 28-day run length was motivated by resolving multiple harmonic constituents. The assimilation of only M2 harmonic data could therefore have been achieved using an emulator trained using much shorter model runs, as used in the adjoint-based assimilation of M2 data via the gradient-based approach, i.e. 3.5 days. In this case, the computational costs of the gradient-based and MCMC methods for assimilating M2 harmonic data would have been comparable. We also note that, while all simulations were performed in parallel across 16 cores, the emulator training runs could take greater advantage of available computational resources since each training run could be performed concurrently. This is in contrast to the gradient-based optimisation approach, where the model runs for each iteration must clearly be performed sequentially.

For the assimilation of data for a single harmonic constituent, the minimisation of the functional for the assimilation of harmonic data via the adjoint is equivalent to the maximisation of the posterior distribution of the MCMC method. Consequently, we find that the parameter estimates resulting from the two M2based calibration methods are consistent, within the uncertainties estimated by the MCMC method. It is therefore clear that there is more information obtained from the MCMC method than from the adjoint approach; specifically, we obtain the full posterior distribution, and can therefore estimate uncertainties in the estimated friction parameters. We therefore suggest that, for the three-dimensional input parameter space used here, the MCMC approach has the potential to be more computationally efficient, by providing more information than the gradient-based approach, at a similar computational cost. 
Since the computationally expensive component of the MCMC framework is in the generation of the emulator training dataset, the subsequent training and use of emulators is flexible, as demonstrated here by the training of two emulators using different combinations of harmonic constituents. By comparing the use of single and multiple constituents for the definition of the likelihood in the MCMC algorithm, we have demonstrated the reduced uncertainty resulting from the assimilation of a greater volume of data. However, since the number of full model runs required to train an emulator depends strongly on the number of degrees of freedom in the input parameter space (via the '10d' rule (Hristov et al., 2017, Sobol, 2001)), the MCMC approach will not scale well with increased complexity in the friction parameter space. We note that some of the computational expense of training high-dimensional surrogate models can be mitigated by incorporating gradient information derived from an adjoint model (e.g. Han et al. (2017)), but this was beyond the scope of this work. The computational cost of the gradient-based calibration approach, on the other hand, is almost independent of the dimension of the parameter space; the cost of the adjoint model runs does not depend on the number of degrees of freedom in the parameter space, and the number of iterations required for convergence would not change significantly, provided that the response surface of the misfit functional with respect to the input parameters remains well-behaved (i.e. the optimisation problem is well-posed; this essentially requires that the assimilated data are sufficient to constrain the unknown parameters). In this study, we used a three-dimensional parameter space for the unknown Manning coefficient, and have concluded that the MCMC and gradient-based approaches can be applied at similar computational cost. Given the considerations above, we suggest that for a parameter space of four or more dimensions, the computational cost of the MCMC approach would exceed that of the adjoint approach, whereas for two or fewer dimensions, the emulator training would be cheaper than the adjoint approach.

Lastly, we note that the availability of timeseries tide gauge data is typically limited to locations on the coastline, while harmonic data is often also available in open locations (e.g. at oil rigs). While we have not directly investigated the relative information contributed by coastal tide gauges compared with openocean locations, it is likely that assimilating data from a variety of observation location environments (e.g. situated in regions of differing sediment types or hydrodynamic regimes) is useful in constraining unknown parameters. The greater availability of harmonic data, across varied locations, suggests that its assimilation is likely to yield tighter constrains on unknown friction parameters, and/or facilitate model calibration with respect to a greater number of degrees of freedom.

\section{Conclusions}

We have approached the problem of estimating three unknown Manning coefficients, corresponding to three groups of sediment types, in a tidal model of the Bristol Channel and Severn Estuary, comparing two parameter estimation methods. The first approach used a Markov Chain Monte Carlo algorithm via the use of a Gaussian process emulator as a surrogate for the full numerical model, and has been applied to the assimilation of tidal harmonic data from 11 locations within the model domain. The second method uses 
the model's adjoint within a gradient-based method, and has been applied to both the assimilation of tidal harmonic data, and tide gauge timeseries data at five coastal locations within the domain. The results from all of these calibration methods are found to be consistent, within the uncertainty estimates resulting from the MCMC approach.

The adjoint approach using timeseries data is the least computationally expensive approach due to its short assimilation window, but for a general application is dependent on the availability of timeseries data at multiple locations within the same time period. For the three-dimensional parameter space used within this work, we have shown that the assimilation of harmonic data via gradient-based and MCMC approaches can be applied at similar computational cost. Since the MCMC approach provides uncertainties in the resulting parameter estimates, which can be valuable information in solving inverse problems, it is therefore more efficient for our purposes. However, considering a more general parameter estimation problem, the adjoint approach will scale better with increased degrees of freedom in the unknown parameter space, and we suggest that for an input parameter space of four dimensions or more, adjoint methods will be more efficient than an emulator-based MCMC approach, whereas for two dimensions or fewer, the MCMC approach will be more efficient. In general, a choice of calibration method should take into account the dimension of the input parameter space, the availability of data and the computational resources available.

Using the M2-only MCMC calibration result, we suggest a best estimate of the Manning coefficients for our rock, gravel and sand sediment groups to be $0.037 \pm 0.07,0.029 \pm 0.009$ and $0.014 \pm 0.003 \mathrm{~s} \mathrm{~m}^{-1 / 3}$, respectively. These parameters are found to decrease multiple measures of model-observation misfit across two numerical models, which suggests that the calibration has avoided excessively correcting for modelspecific numerical errors, and therefore that these parameter estimates are physically meaningful.

\section{Acknowledgements}

SCW's work was funded through the EPSRC Centre for Doctoral Training in Fluid Dynamics across Scales (Grant EP/L016230/1). MDP would additionally like to acknowledge EPSRC support under Grant EP/R029423/1. We also acknowledge the Research Computing Service at Imperial College London for access to computing resources. This study uses data from the National Tidal and Sea Level Facility, provided by the British Oceanographic Data Centre and funded by the Environment Agency.

\section{References}

G. J. Arcement and V. R. Schneider. Guide for Selecting Manning's Roughness Coefficients for Natural Channels and Flood Plains. Technical report, 1989.

A. Avdis, A. S. Candy, J. Hill, S. C. Kramer, and M. D. Piggott. Efficient unstructured mesh generation for marine renewable energy applications. Renewable Energy, 116:842-856, 2018. ISSN 0960-1481. doi: https: 
//doi.org/10.1016/j.renene.2017.09.058. URL http://www.sciencedirect.com/science/article/pii/ S0960148117309205.

C. Bischof, A. Carle, G. Corliss, A. Griewank, and P. Hovland. ADIFOR-Generating Derivative Codes from Fortran Programs. Scientific Programming, 1:11-29, 1992.

H. Chen, A. Cao, J. Zhang, C. Miao, and X. Lv. Estimation of spatially varying open boundary conditions for a numerical internal tidal model with adjoint method. Mathematics and Computers in Simulation, 97:14-38, mar 2014. ISSN 0378-4754. doi: 10.1016/J.MATCOM.2013.08.005. URL https://www.sciencedirect.com/science/article/pii/S0378475413002000.

Digimap. Marine Themes Digital Elevation Model 1 Arc Second [ASC geospatial data], Scale 1:50000, Tiles: 5050510045, 5050510050, 5051010030, 5051010035, 5051010040, 5051010045, 5051010050, 5051510025, 5051510030, 5051510035, 5051510040, 5051510045, 5051510050, Updated: 9 September 2016, OceanWise, Using: EDINA Marine Digimap Service, https://digimap.edina.ac.uk, 2016.

G. D. Egbert and S. Y. Erofeeva. Efficient inverse modeling of barotropic ocean tides. Journal of Atmospheric and Oceanic Technology, 19(2):183-204, 2002. ISSN 07390572. doi: 10.1175/1520-0426(2002)019<0183: EIMOBO $\rangle 2.0 . \mathrm{CO} ; 2$.

G. Evensen. The Ensemble Kalman Filter for Combined State and Parameter Estimation. IEEE Control Systems, 29(3):83-104, 2009.

S. W. Funke. The automation of PDE-constrained optimisation and its applications. PhD thesis, Imperial College London, 2012.

S. W. Funke, S. C. Kramer, and M. D. Piggott. Design optimisation and resource assessment for tidal-stream renewable energy farms using a new continuous turbine approach. Renewable Energy, 99:1046-1061, 2016. ISSN 0960-1481. doi: 10.1016/j.renene.2016.07.039. URL http://dx.doi.org/10.1016/j.renene. 2016. 07.039.

C. Geuzaine and J. F. Remacle. Gmsh: A 3-D finite element mesh generator with built-in pre- and postprocessing facilities. International Journal for Numerical Methods in Engineering, 79(11):1309-1331, 2009. ISSN 00295981. doi: 10.1002/nme.2579.

R. Giering and T. Kaminski. Recipes for Adjoint Code Construction. ACM Transactions on Mathematical Software, 24(4):437-474, 1998. ISSN 00983500. doi: 10.1145/293686.293695.

GPy. GPy: A gaussian process framework in python. http://github.com/SheffieldML/GPy, since 2012.

L. Graham, T. Butler, S. Walsh, C. Dawson, and J. J. Westerink. A Measure-Theoretic Algorithm for Estimating Bottom Friction in a Coastal Inlet: Case Study of Bay St. Louis during Hurricane Gustav (2008). Monthly Weather Review, 145:929-954, 2017. ISSN 0027-0644. doi: 10.1175/mwr-d-16-0149.1. 
A. Griewank and A. Walther. Evaluating derivatives: principles and techniques of algorithmic differentiation. Society for Industrial and Applied Mathematics, 2nd edition, 2008.

N. Guillou and J. Thiébot. The impact of seabed rock roughness on tidal stream power extraction. Energy, 112:762-773, 2016. ISSN 03605442. doi: 10.1016/j.energy.2016.06.053.

H. Haario, E. Saksman, and J. Tamminen. An adaptive Metropolis algorithm. Bernoulli, 7(2):223-242, 2001. ISSN 13507265. doi: 10.2307/3318737.

J. W. Hall, L. J. Manning, and R. K. Hankin. Bayesian calibration of a flood inundation model using spatial data. Water Resources Research, 47(5):1-14, 2011. ISSN 00431397. doi: 10.1029/2009WR008541.

Z.-H. Han, Y. Zhang, C.-X. Song, and K.-s. Zhang. Weighted Gradient-Enhanced Kriging for HighDimensional Surrogate Modeling and Design Optimization. AIAA, 55(12), 2017. doi: 10.2514/1.J055842.

W. K. Hastings. Monte Carlo sampling methods using Markov chains and their applications. 1970.

A. W. Heemink, E. E. A. Mouthaan, M. R. T. Roest, E. A. H. Vollebregt, K. B. Robaczewska, and M. Verlaan. Inverse 3D shallow water flow modelling of the continental shelf. Continental Shelf Research, 22:465-484, 2002.

J.-M. Hervouet. Hydrodynamics of free surface flows: modelling with the finite element method. Wiley, 2007.

K. J. Horsburgh and C. Wilson. Tide-surge interaction and its role in the distribution of surge residuals in the North Sea. Journal of Geophysical Research, 112(C8), 2007. ISSN 21699291. doi: 10.1029/2006JC004033.

P. O. Hristov, F. A. DiazDelaO, E. I. Saavedra Flores, C. F. Guzmán, and U. Farooq. Probabilistic sensitivity analysis to understand the influence of micromechanical properties of wood on its macroscopic response. Composite Structures, 2017. ISSN 02638223. doi: 10.1016/j.compstruct.2017.08.105.

T. Kärnä, B. de Brye, O. Gourgue, J. Lambrechts, R. Comblen, V. Legat, and E. Deleersnijder. A fully implicit wetting-drying method for DG-FEM shallow water models, with an application to the Scheldt Estuary. Computer Methods in Applied Mechanics and Engineering, 200(5-8):509-524, 2011. ISSN 00457825. doi: 10.1016/j.cma.2010.07.001.

T. Kärnä, S. C. Kramer, L. Mitchell, D. A. Ham, M. D. Piggott, and A. M. Baptista. Thetis coastal ocean model: Discontinuous Galerkin discretization for the three-dimensional hydrostatic equations. Geoscientific Model Development, 11(11):4359-4382, 2018. ISSN 19919603. doi: 10.5194/gmd-11-4359-2018.

P. Lang, J. Desombre, R. Ata, C. Goeury, and J. M. Hervouet. User manual of opensource software TELEMAC-2D. Report, EDF-R\&D, www.opentelemac.org, 2014. V7.

$\mathrm{X}$. Lu and J. Zhang. Numerical study on spatially varying bottom friction coefficient of a 2D tidal model with adjoint method. Continental Shelf Research, 26(16):1905-1923, oct 2006. ISSN 0278-4343. doi: 10.1016/J. CSR.2006.06.007. URL https://www.sciencedirect.com/science/article/pii/S027843430600210X. 
J. Marotzke, R. Giering, K. Q. Zhang, D. Stammer, C. Hill, and T. Lee. Construction of the adjoint MIT ocean general circulation model and application to Atlantic heat transport sensitivity. Journal of Geophysical Research, 104:29529-29547, 1999. doi: 10.1029/1999JC900236.

S. Maßmann. Tides on unstructured meshes. PhD thesis, Universitat Bremen, 2010a.

S. Maßmann. Sensitivities of an adjoint, unstructured mesh, tidal model on the European Continental Shelf. Ocean Dynamics, 60(6):1463-1477, dec 2010b. ISSN 16167341. doi: 10.1007/s10236-010-0347-6. URL https://doi.org/10.1007/s10236-010-0347-6.

T. Mayo, T. Butler, C. Dawson, and I. Hoteit. Data assimilation within the Advanced Circulation (ADCIRC) modeling framework for the estimation of Manning's friction coefficient. Ocean Modelling, 76:43-58, 2014. ISSN 14635003. doi: 10.1016/j.ocemod.2014.01.001. URL http://dx.doi.org/10.1016/j . ocemod. 2014. 01.001.

S. K. Mitusch, S. W. Funke, and J. S. Dokken. dolfin-adjoint 2018.1: automated adjoints for FEniCS and Firedrake. Journal of Open Source Software, 4(38), 2019.

S. P. Neill, A. Angeloudis, P. E. Robins, I. Walkington, S. L. Ward, I. Masters, M. J. Lewis, M. Piano, A. Avdis, M. D. Piggott, G. Aggidis, P. Evans, T. A. Adcock, A. Židonis, R. Ahmadian, and R. Falconer. Tidal range energy resource and optimization Past perspectives and future challenges. Renewable Energy, 127:763-778, 2018. ISSN 18790682. doi: 10.1016/j.renene.2018.05.007.

J. Pall, R. Chandra, D. Azam, T. Salles, J. M. Webster, and S. Cripps. BayesReef: A Bayesian inference framework for modelling reef growth in response to environmental change and biological dynamics. 2018. URL http://arxiv.org/abs/1808.02763.

F. Rathgeber, D. A. Ham, L. Mitchell, M. Lange, F. Luporini, A. T. McRae, G. T. Bercea, G. R. Markall, and P. H. Kelly. Firedrake: Automating the finite element method by composing abstractions. ACM Transactions on Mathematical Software, 43(3), 2016. ISSN 15577295. doi: 10.1145/2998441.

P. Schureman. Manual of harmonic analysis and prediction of tides. Number 98. US Government Printing Office, 1941.

SHOM. https://data.shom.fr, 2019.

D. Sivia and J. Skilling. Data analysis: a Bayesian tutorial. OUP Oxford, 2006.

I. M. Sobol. Global sensitivity indices for nonlinear mathematical models and their Monte Carlo estimates. Mathematics and Computers in Simulation, 2001. ISSN 03784754. doi: 10.1016/S0378-4754(00)00270-6.

I. Sraj, M. Iskandarani, A. Srinivasan, W. C. Thacker, J. Winokur, A. Alexanderian, C. Y. Lee, S. S. Chen, and O. M. Knio. Bayesian inference of drag parameters using AXBT data from typhoon fanapi. Monthly Weather Review, 141(7):2347-2367, 2013. ISSN 00270644. doi: 10.1175/MWR-D-12-00228.1. 
I. Sraj, M. Iskandarani, W. Carlisle Thacker, A. Srinivasan, and O. M. Knio. Drag parameter estimation using gradients and hessian from a polynomial chaos model surrogate. Monthly Weather Review, 142(2): 933-941, 2014a. ISSN 00270644. doi: 10.1175/MWR-D-13-00087.1.

I. Sraj, K. T. Mandli, O. M. Knio, C. N. Dawson, and I. Hoteit. Uncertainty quantification and inference of Manning's friction coefficients using DART buoy data during the Thoku tsunami. Ocean Modelling, 83: 82-97, 2014b. ISSN 14635003. doi: 10.1016/j.ocemod.2014.09.001. URL http://dx.doi.org/10.1016/ j.ocemod.2014.09.001.

P. M. Tagade, B. M. Jeong, and H. L. Choi. A Gaussian process emulator approach for rapid contaminant characterization with an integrated multizone-CFD model. Building and Environment, 2013. ISSN 03601323. doi: 10.1016/j.buildenv.2013.08.023.

M. H. Tber, L. Hascoët, A. Vidard, and B. Dauvergne. Building the Tangent and Adjoint codes of the Ocean General Circulation Model OPA with the Automatic Differentiation tool TAPENADE, 2007.

C. V. Vouriot, A. Angeloudis, S. C. Kramer, and M. D. Piggott. Fate of large-scale vortices in idealized tidal lagoons. Environmental Fluid Mechanics, 19(2):329-348, apr 2019. ISSN 15731510. doi: 10.1007/ s10652-018-9626-4. URL https://doi.org/10.1007/s10652-018-9626-4.

S. C. Warder, K. J. Horsburgh, and M. D. Piggott. Adjoint-based sensitivity analysis for a numerical storm surge model. Ocean Modelling, Submitted 2020.

C. Wilson, K. J. Horsburgh, J. Williams, J. Flowerdew, and L. Zanna. Tide-surge adjoint modeling: A new technique to understand forecast uncertainty. Journal of Geophysical Research: Oceans, 118(10): 5092-5108, 2013. ISSN 21699291. doi: 10.1002/jgrc.20364.

D. Xie, Z. Wang, S. Gao, and H. J. De Vriend. Modeling the tidal channel morphodynamics in a macro-tidal embayment, Hangzhou Bay, China. Continental Shelf Research, 2009. ISSN 02784343. doi: 10.1016/j.csr. 2009.03.009.

J. Zhang, X. Lu, P. Wang, and Y. P. Wang. Study on linear and nonlinear bottom friction parameterizations for regional tidal models using data assimilation. Continental Shelf Research, 31(6):555-573, apr 2011. ISSN 0278-4343. doi: 10.1016/J.CSR.2010.12.011. URL https://www.sciencedirect.com/science/ article/pii/S0278434310003857.

C. Zhu, R. H. Byrd, P. Lu, and J. Nocedal. Algorithm 778: L-BFGS-B: Fortran subroutines for large-scale bound-constrained optimization. ACM Transactions on Mathematical Software (TOMS), 23(4):550-560, 1997. 